UTED Seec

\title{
LA FORMAZIONE INIZIALE DEGLI INSEGNANTI IN ITALIA
}

\section{Initial teacher education in Italy}

\author{
Carlo Cappa, Orazio Niceforo y Donatella Palomba *
}

\section{RESUMEN}

L'articolo è diviso in tre parti. La prima presenta gli elementi storici e di sfondo che occorre tenere presenti per comprendere l'evoluzione della formazione iniziale degli insegnanti verso gli attuali modelli, che pur essendo oggi tutti gestiti dalle università conservano una sensibile diversità a seconda che si tratti di formare maestri per la scuola dell'infanzia e primaria oppure professori di scuola secondaria di primo e secondo grado. La seconda parte analizza il passaggio, maturato tra la fine del secolo scorso e l'inizio del XXI secolo, della formazione dei maestri dalla scuola secondaria (Istituto magistrale) all'università (Corso di laurea in Scienze della formazione primaria), e l'istituzione delle Scuole di Specializzazione per l'Insegnamento Secondario (SSIS) fino alla soppressione di queste ultime, avvenuta nel 2009. La terza parte presenta il quadro attuale della formazione iniziale dei docenti in Italia con riguardo soprattutto agli insegnanti della scuola secondaria, per i quali - a differenza che per i maestri - sono intervenute dal 2010 importanti innovazioni legislative. Le SSIS sono state infatti sostituite con il Tirocinio Formativo Attivo (TFA), attualmente in fase di attuazione da parte delle università. Nella parte finale dell'articolo si traccia un primo bilancio dell'esperienza del TFA e si indicano alcuni problemi aperti. Il focus rimane sostanzialmente sulla formazione iniziale, in quanto in Italia la formazione in servizio, che pure non è del tutto assente, non è mai stata oggetto di una normativa né di una azione organica, ma avviene, quando avviene, sulla base di iniziative di specifici gruppi o associazioni.

\footnotetext{
"Università degli Studi di Roma "Tor Vergata" (Italy).
} 
PALABRAS CLAVE: Formazione degli insegnanti; Formazione iniziale; Tirocinio; Università; Scuola; Italia.

\section{ABSTRACT}

The article is organised in three parts. The first one illustrates the historical features and the institutional and political background that have to be taken into account in order to understand how initial teacher education and training has developed in Italy. Presently, all teacher education is managed by Universities, but a considerable difference still remains between the organisation of courses for primary and pre-primary teachers, on one hand, and secondary teachers, on the other. The second part analyses the transition, between the end of the $20^{\text {th }}$ century and the beginning of the $21^{\text {st }}$, from an education for primary teachers that took place at high school level (Istituto Magistrale) to a fully University one (Corso di laurea in Scienze della formazione primaria); and it also examines the crucial issue of the creation, in the same period, of the Post-Graduate Schools for Secondary Teachers (Scuole di Specializzazione per l'Insegnamento Secondario - SSIS), until they were abolished in 2009. The third part shows the present framework of initial teacher training in Italy, mainly related to teaching in lower and upper secondary schools. At this level of schooling the legislation was recently changed; the Scuole di Specializzazione per l'Insegnamento Secondario (SSIS) have been replaced with the Tirocinio Formativo Attivo (TFA), which at present is being implemented for the first time by the universities. In the final section of this part a provisional balance of the innovation is outlined and some critical issues are pointed out. The essay is essentially focused on initial teacher training, as in Italy in-service training, while not completely absent, has never been the object of specific regulation nor of systematic actions, but it exists when it exists - on the basis of the initiative of individual groups or associations.

KEY WORDS: Teacher education; Initial teacher training; Teaching practice, University; School; Italy.

$* * * * *$

\section{INTRODUCCIÓN}

Nel momento in cui questo lavoro viene scritto, la formazione degli insegnanti in Italia si trova in una fase particolarmente delicata, specie in relazione alla formazione degli insegnanti secondari. Sta infatti giungendo a conclusione il primo anno accademico di parziale realizzazione di un provvedimento di riforma che, approvato nel 2010 secondo uno schema che si voleva organico, è stato poi realizzato in modo graduale: a partire dal 2011-2012 si è data attuazione a quanto previsto per gli insegnanti di scuola primaria e materna, mentre per la scuola secondaria una effettiva attuazione di una parte dei provvedimenti previsti si è avuta solo a partire dal 2012-2013. Ci si trova dunque ancora in medias res, il che rende difficile compiere un vero e proprio bilancio.

Questi provvedimenti si inseriscono tuttavia su uno sfondo la cui conoscenza è necessaria per comprendere i tratti fondamentali delle innovazioni che essi introducono. Il presente saggio 
cercherà dunque di tracciare un quadro che, per quanto sintetico, sia sufficientemente articolato e approfondito, e che riesca a mettere in evidenza le linee essenziali e le caratteristiche di base della formazione degli insegnanti in Italia secondo le tendenze principali emerse a partire dalla seconda metà del Novecento, fino a giungere ad una descrizione della situazione attuale. In particolare, dopo una prima parte di inquadramento generale, la seconda parte si soffermerà sulla travagliata stagione di riforme che la formazione degli insegnanti, insieme a tanti altri ambiti del sistema formativo e non solo, ha vissuto nel periodo a cavallo fra il XX e il XXI secolo, mentre la terza darà più dettagliatamente conto dei caratteri principali della riforma ora in atto ${ }^{1}$.

\section{IL QUADRO DI SFONDO}

\subsection{La figura dell'insegnante tra centralità e contraddizioni}

Una descrizione e un'analisi delle vicende relative alla formazione degli insegnanti in Italia richiedono di muoversi fra diverse polarità, che esistono per lo più anche in altri paesi ma si presentano qui in modo diverso e talvolta più accentuato, divenendo vere e proprie dicotomie, come accade per quella fra formazione professionale e formazione disciplinare, che si sta da anni faticosamente cercando di ricomporre, e per quella che si riscontra fra la formazione degli insegnanti di scuola primaria e quella degli insegnanti di scuola secondaria, che rimane invece del tutto confermata anche nei più recenti provvedimenti. Si deve poi segnalare, peculiarità tutta italiana, una notevole discrasia fra formazione e reclutamento, che affonda le sue radici in ragioni storiche, ma che ha finito per produrre una impasse difficilissima da superare ancora oggi. A queste va aggiunto il diuturno braccio di ferro fra lo Stato italiano e la Chiesa cattolica per l'influenza sul sistema educativo, che ha avuto un notevole impatto sulla politica dell'istruzione nel nostro paese, e di cui la formazione degli insegnanti costituisce evidentemente uno dei nodi cruciali. Si dice talvolta che le mancate riforme e le carenze nella formazione degli insegnanti siano la prova del fatto che l'importanza della figura dell'insegnante sia sottovalutata nel contesto italiano; ma si può - e forse si deve - invece avanzare anche l'interpretazione opposta, ovvero che sia proprio il riconoscimento della centralità della figura dell'insegnante a rendere così difficile il superamento delle diverse concezioni che se ne hanno da diverse parti ideologiche e politiche, e la convergenza su un percorso formativo concordato.

In realtà, la concezione della figura dell'insegnante segue tutte le complesse vicende che hanno segnato la scuola italiana dall'Unità ad oggi, riflettendone non solo i problemi, ma soprattutto le incertezze e le controversie rispetto ad alcuni caratteri fondamentali dell'educazione che si doveva offrire alle giovani generazioni. Tali controversie rispecchiano fra l'altro la difficoltà di trovare un consenso sociale che attraversi le "diverse appartenenze" che hanno segnato la società italiana a partire dal dopoguerra, e si fanno particolarmente accentuate via via che ci si allontana dall'istruzione di base (PALOMBA, 1988, 2009); e questa difficoltà incide fortemente sulla concezione stessa del ruolo della scuola nel suo duplice aspetto di luogo di trasmissione culturale e al contempo di trasformazione, che inevitabilmente riflette in qualche modo la società in cui opera,

\footnotetext{
${ }^{1}$ Gli autori hanno discusso insieme impostazione e contenuti del lavoro, ma ciascuno ha approfondito una delle parti e ne ha curato la stesura. Specificamente, la parte prima è stata scritta da Donatella Palomba, la seconda da Carlo Cappa e la terza da Orazio Niceforo.
} 
ma deve allo stesso tempo prepararne il futuro. Evidentemente il ruolo dell'insegnante sarà pensato in modo ben diverso a seconda di come ci si colloca nella gamma delle posizioni possibili nell'arco che va da una stretta conservazione al più radicale rinnovamento. Si pensi ad un dibattito, assai vivo nei decenni centrali del XX secolo, che la dice lunga sulle diversità di concezione relativamente alla professionalità insegnante, ovvero il dibattito che opponeva la "vocazione" all'insegnamento rispetto alla formazione professionale specifica. E' facile osservare che lo stesso significato etimologico dei termini "vocazione" e "professione" riflette appunto questa tensione fra la "chiamata" di natura spirituale e la professione nel suo aspetto operativo (tensione particolarmente evidente nella lingua inglese e in quella tedesca, dove i termini si sovrappongono) - ma proprio per questo la controversia sul modo in cui si scioglie questo nodo è rivelatrice della distanza fra diverse concezioni del ruolo insegnante.

Significativamente, nel cruciale decennio degli anni Sessanta del Novecento, quando il ruolo del sistema di istruzione nel suo insieme era visto per un verso come quello di un apparato ideologico di Stato (Althusser) o quello della "riproduzione" (Bourdieu), e per altro verso come il luogo privilegiato da cui far partire, se non la rivoluzione, almeno delle radicali "riforme di struttura", si identificava in un certo senso nel docente il perno del sistema, specie nella scuola secondaria. Non a caso il bruciante piccolo libro della Scuola di Barbiana fondata da Don Milani con la sua sferzante denuncia del carattere di classe della scuola - si intitolava Lettera a una professoressa (SCUOLA DI BARBIANA, 1967); quella stessa "professoressa" che due anni più tardi verrà definita "vestale della classe media" nel celebre titolo di un altro volume, che raccoglieva i risultati di una indagine sociologica che si soffermava sugli atteggiamenti degli insegnanti rispetto all'innovazione, mettendone in luce le resistenze, specie in relazione alla riforma della scuola media, alla cui forte portata innovatrice faremo cenno fra breve (BARBAGLI e DEI, $1969)^{2}$.

In quegli stessi anni Sessanta, ma su un altro versante, analoga centralità si riconosceva al ruolo insegnante da parte di chi si occupava di tracciare le linee di riforma del sistema scolastico, in una stagione riformista - quella che portò al primo governo di centro-sinistra - che tentava di elaborare una programmazione scolastica all'interno di una più generale politica di piano. Come ricorda Giunio Luzzatto (LUZZATTO, 2011a), nel 1963 la relazione conclusiva della Commissione parlamentare di indagine sulla scuola metteva al primo punto l'esigenza di interventi relativi all'università, mirando specificamente ed esplicitamente al suo ruolo nella formazione degli insegnanti. Ma appunto, è qui che la complessità della situazione e la difficoltà di raggiungere un consenso di base si fece particolarmente sentire, provocando quello stallo da cui non si sarebbe usciti se non dopo circa tre decenni.

In particolare, rimase viva la dicotomia fra i percorsi di formazione degli insegnanti di scuola materna ed elementare, da un lato, e quelli di scuola secondaria, dall'altro. Tradizionalmente, e fino alla fine degli anni Novanta del XX secolo, in Italia l'insegnante elementare - il maestro era formato a livello di scuola secondaria, con la frequenza dell'Istituto magistrale, della durata di

\footnotetext{
${ }^{2}$ È da segnalare peraltro che la questione degli atteggiamenti degli insegnanti rimane centrale ancora oggi, se è vero che anche in indagini molto più recenti si riscontra, a fronte di un'apertura dichiarata all'innovazione, una molto maggior resistenza alla loro applicazione pratica (VANNINI, 2012).
} 
quattro anni, che includeva nel curriculum alcune materie di "scienze dell'educazione", in particolare pedagogia e psicologia, e conduceva a un diploma abilitante. Non si prevedeva dunque una formazione a livello di insegnamento superiore: anche se un decreto del $1974^{3}$ richiedeva formalmente per tutti i livelli scolastici «una formazione universitaria completa da conseguire presso le università od altri istituti di istruzione superiore», tuttavia relativamente alla formazione dei maestri elementari tale prescrizione non fu attuata.

Situazione esattamente opposta si riscontrava nel caso della scuola secondaria, sia inferiore ("scuola media") che superiore, dove per insegnare qualsiasi disciplina era necessario aver conseguito una laurea universitaria, ma non era prevista nessuna specifica preparazione professionale: il concorso per l'accesso al ruolo insegnante si svolgeva per titoli e per esami di natura sostanzialmente disciplinare. La formazione superiore per gli insegnanti elementari si limitò dunque ad una affermazione di principio rimasta sulla carta, mentre la formazione professionale degli insegnanti di scuola secondaria non era neppure menzionata.

L'inadeguatezza di tale impostazione, che su ciascuno dei due versanti mancava dell'uno o dell'altro degli elementi essenziali per una formazione docente completa, era ben presente nei dibattiti e nei documenti che furono prodotti lungo l'arco degli anni, ma si dovette attendere appunto gli anni Novanta per vedere prima l'istituzione e poi l'attuazione delle relative strutture.

Tanto più stridente era il permanere di tale dicotomia in quanto la stagione delle riforme non era rimasta senza esiti in altri settori, e in particolare aveva dato vita nel 1962 a quella legge di riforma della scuola media ${ }^{4}$ che condusse alla creazione di quello che in quel momento era il sistema di base più unitario dell'intero panorama europeo, unificando i canali esistenti nel primo triennio successivo alla scuola elementare in un ciclo del tutto unitario - la "scuola media unica", appunto.

Il sistema scolastico italiano si configurava dunque secondo una articolazione che comprendeva, dopo una istruzione prescolare non obbligatoria, una scuola elementare quinquennale, una scuola media inferiore unificata di durata triennale, e una scuola secondaria superiore, di durata quinquennale, divisa in diversi canali. Il ciclo dell'obbligo in quel momento comprendeva i primi otto anni di scolarità, che non avendo differenziazioni interne costituivano in un certo senso un ciclo unico, anche se, a segnarne comunque la separazione, rimaneva in vigore un esame per l'ammissione alla scuola media.

L'aspetto unitario si è ulteriormente accentuato in tempi recenti, con l'abolizione di tale esame, e la comparsa, seppur non generalizzata, degli istituti comprensivi, che accorpano istituti di istruzione primaria e secondaria inferiore. Tuttavia, benché si possa dire che ormai da un cinquantennio vi sia nel sistema scolastico italiano un ciclo che si può considerare unitario che va dalla prima alla ottava classe di scuola, l'articolazione in scuola primaria e scuola secondaria

\footnotetext{
${ }^{3}$ Decreto del Presidente Repubblica 31 maggio 1974, n. 417, Norme sullo stato giuridico del personale docente, direttivo ed ispettivo della scuola materna, elementare, secondaria ed artistica dello Stato.

${ }^{4}$ Legge 31 dicembre 1962, n. 1859, Istituzione e ordinamento della scuola media statale.
} 
permane: ed è a questa che si conforma la preparazione degli insegnanti, in quanto, pur nelle varie trasformazioni che si sono avute, comprese le più recenti, rimangono del tutto distinti i corsi per la formazione degli insegnanti di scuola materna e primaria, e quelli della scuola secondaria, a partire dal primo anno della scuola secondaria inferiore, ovvero il sesto anno di scolarità.

\subsection{Gli anni Settanta e Ottanta: dibattiti e sperimentazioni}

Come abbiamo detto, si sono dovuti attendere gli anni Novanta per avere prima l'istituzione e successivamente l'attuazione di provvedimenti di riforma della formazione insegnanti.

Tuttavia non tutto era fermo, anzi: da un lato, erano sempre più numerosi i maestri che, di propria iniziativa, proseguivano gli studi a livello universitario. Dall'altro, fiorivano iniziative di gruppi in cui docenti di scuola secondaria e specialisti universitari collaboravano sperimentando procedure e modelli di formazione, che costituivano punti di riferimento per molti dei più motivati operatori della scuola. Anche qui compare, se non proprio una dicotomia, certo una qualche sfasatura fra due diversi ambiti, in quanto tali attività coinvolgevano, oltre naturalmente agli specialisti di scienze dell'educazione, prevalentemente docenti universitari delle aree scientifiche. Questo è particolarmente evidente per la matematica, seguendo una tradizione italiana dalle radici antiche, che risale almeno a Giuseppe Peano e Lucio Lombardo Radice (non a caso ancora oggi due degli accademici più impegnati nelle problematiche dell'insegnamento secondario, sia pur su fronti fieramente opposti, sono due matematici, Giunio Luzzatto e Giorgio Israel); ma più in generale furono gli accademici di ambito scientifico a mostrare maggior coinvolgimento nelle questioni didattiche e di formazione insegnanti, mentre l'ambito umanistico, dove pure non mancavano docenti interessati, tendeva nel suo complesso a privilegiare l'aspetto disciplinare.

Si trattava comunque di formazione continua (peraltro non normata nella legislazione italiana), e non di formazione iniziale: ma poneva le basi per i modelli che furono successivamente elaborati. Nel 1978, usciva poi un lavoro di Aldo Visalberghi (VISALBERGHI, 1978) destinato ad avere un notevole impatto sul futuro impianto della struttura della formazione, in cui si individuavano le componenti che dovevano concorrere a formare le competenze del docente e se ne argomentavano le articolazioni e le caratteristiche, secondo una "enciclopedia pedagogica" che vedeva la presenza di un settore dei contenuti, di un settore psicologico, di un settore sociologico e di un settore metodologico-didattico.

Come si vede, e si vedrà meglio nelle sezioni successive, vi è un deciso scarto non solo fra l'elaborazione teorica e l'impegno di alcuni, da un lato, e la realizzazione pratica, dall'altro, ma anche fra l'approvazione di normative che fanno affermazioni di principio o istituiscono corsi di formazione e i tempi e i modi della loro effettiva applicazione.

Questo denuncia la complessità della situazione, che a nostro avviso rimanda a tutte le tensioni che abbiamo menzionato, e in particolare alla mancata convergenza su una concezione comune del ruolo della scuola, e di conseguenza della figura docente. Tuttavia questo scarto, e lo stallo che ne deriva, produce anche un circolo vizioso, aggravando quello che rimane a tutt'oggi il 
maggiore ostacolo concreto per un funzionamento efficace della preparazione dei docenti in Italia, ovvero la discontinuità fra formazione e reclutamento.

In effetti, il protrarsi della non-riforma ha fatto sì che un numero crescente di diplomati $\mathrm{e}$ laureati iniziasse ad insegnare secondo modalità considerate più o meno provvisorie, andando a formare un corpo sempre più numeroso di docenti a volte precari, a volte stabilizzati secondo diverse procedure, i cui diritti acquisiti nel tempo vanno poi a scontrarsi con le possibilità di inserimento di giovani leve fornite di quella preparazione più avanzata prevista dai provvedimenti che infine hanno cominciato ad essere attuati a partire dalla fine degli anni Novanta.

Nel 1998-99 infatti ebbe inizio finalmente l'attuazione del corso di laurea per maestri, e l'anno successivo quella delle Scuole di Specializzazione all'Insegnamento Secondario (SSIS). Sembravano dunque saldarsi le due componenti - accademica e professionale - della formazione del personale docente, dando infine attuazione, oltre vent'anni dopo, al principio affermato nel Decreto del 1974. Come verrà illustrato e approfondito nelle pagine che seguono, in realtà le vicende saranno assai travagliate, in particolare per quel che riguarda le scuole secondarie - e le possibili ragioni ne verranno attentamente esaminate.

Prima di concludere questo breve quadro di sfondo, tuttavia, vorremmo ancora dire una parola su quella stagione delle riforme che il sistema di istruzione italiano visse negli anni cruciali di fine secolo - fine millennio, in realtà - di cui ha fatto parte l'attuazione dei provvedimenti sopra menzionati, e che presenta alcune caratteristiche che forse possono contribuire a spiegare alcuni dei problemi successivamente incontrati. Nel 1996 l'Italia ebbe per la prima volta un governo di cui facevano parte a pieno titolo ministri provenienti da quell'area ex comunista che era stata tenuta fuori dalle stanze del potere per la conventio ad excludendum vigente fino alla caduta del muro di Berlino. E' più che comprensibile che i ministri di questo nuovo governo avessero una visione riformista molto radicale, e che riprendessero quindi, con spirito nuovo e adatto ai tempi e con una assai più spiccata sensibilità europea, quell'approccio delle "riforme di struttura" che già si era espresso nei decenni precedenti. In particolare, il ministro Berlinguer, ex rettore dell'Università di Siena, uno dei promotori a livello europeo di quella Dichiarazione della Sorbona che segnerà il primo momento del Processo di Bologna, aveva una visione globale dei problemi dell'istruzione che lo chiamava ad una azione complessiva sui vari settori di essa (BERLINGUER, 2001). Una così vasta opera di riforma, tuttavia, poneva nella pratica non indifferenti problemi di raccordo fra le diverse innovazioni, creando situazioni a volte difficili da gestire e ritardi e sfasature nei tempi e nei modi di attuazione. A titolo di esempio si può possono citare proprio le SSIS, che prevedevano per la formazione degli insegnanti secondari un corso biennale dopo la laurea. Nel momento in cui il provvedimento fu attuato, la grandissima maggioranza dei corsi di laurea aveva una durata di quattro anni; ma già dall'anno successivo una nuova legge istituiva una nuova struttura universitaria, che seguiva il modello cosiddetto "di Bologna", ed era dunque articolata in un corso di laurea di primo livello, di durata triennale, ed uno di secondo livello, biennale. In pratica, questo voleva dire portare a sette anni il percorso di formazione degli insegnanti, contro i quattro della precedente laurea disciplinare. Invero, molti (in particolare nelle aree scientifiche) sostenevano che la laurea triennale potesse essere sufficiente per l'accesso alla SSIS; si rimase a lungo nell'incertezza, ma infine la riserva fu sciolta nel senso di richiedere la laurea di secondo livello, 
con un percorso complessivo davvero assai lungo. Non fu certo solo questo a causare difficoltà alle SSIS; ma l'esempio è significativo di alcune delle difficoltà conseguenti all'attività riformatrice - in alcuni casi febbrile - di quel periodo, che certamente favorirono poi l'opera di cancellazione o modifica da parte dei successivi governi conservatori di molte delle riforme approvate.

E' in questa situazione complessa, dunque, che si innestano le vicende, e le crisi, che verranno meglio analizzate nelle pagine a venire.

2. DALLA FINE DEL VENTESIMO SECOLO AL PRIMO DECENNIO DEL XXI SECOLO: MATURAZIONE E INCERTO COMPIMENTO DI UN LUNGO PERCORSO

Il profilo della professionalità docente, che si è lentamente delineato in Italia nell'arco di più di un secolo di storia della scuola, ha trovato una parziale sistemazione nei provvedimenti legislativi degli anni Novanta del secolo scorso. Essi hanno recepito e, in parte, fissato un ricco dibattito, che fece tesoro anche di una vivace attività di sperimentazione avviata all'interno dell'università. Naturalmente, non erano certo mancati in precedenza tentativi di affrontare il delicato nodo della formazione iniziale degli insegnanti (SANTONI RUGIU e SANTAMAITA, 2011, pp. 165-167; LUZZATTO, 2001), ma queste episodiche prove, che in alcuni casi hanno coinvolto personalità di alto profilo molto sensibili a tale tema, come ad esempio studiosi appartenenti all'area della matematica (LUZZATTO, 2001: 17-21), non giunsero mai a fornire una risposta soddisfacente e complessiva alla sempre più pressante richiesta di un superamento tanto della differenza dei livelli delle istituzioni educative a cui erano affidati i percorsi d'istruzione secondo il grado della scuola nel quale si sarebbe andato a insegnare, quanto della schiacciante superiorità della preparazione disciplinare rispetto a quella professionale (BELLATALLA, 2009: 90).

La legge che provò a fronteggiare questa incerta situazione, recependo le numerose sollecitazioni provenienti dal mondo della scuola e dall'intensa produzione scientifica sulla formazione insegnanti, fu la n. 341 del 1990, dal titolo Riforma degli ordinamenti didattici universitari. Come si può agevolmente comprendere dalla denominazione di questa legge, il suo obiettivo non si limitava certo a una modifica della figura docente, giacché essa investiva in maniera più ampia l'intera istruzione superiore, proponendo profonde novità, a testimonianza di un fermento che, seppur non sempre foriero di soluzioni pienamente soddisfacenti, incise in modo non superficiale nel profilo dell'università in Italia. La legge istituì sia il corso di laurea in Scienze della formazione primaria sia la Scuola di specializzazione per l'insegnamento secondario (SSIS) ${ }^{5}$. I due nuovi percorsi collocavano risolutamente la formazione degli insegnanti in ambito universitario, ma hanno avuto storie differenti, innestate nelle tante trasformazioni che l'istruzione superiore ha attraversato negli ultimi vent'anni; queste trasformazioni hanno assunto, in alcuni casi ${ }^{6}$, le

\footnotetext{
${ }^{5}$ Come si può osservare, nonostante le importanti innovazioni, i percorsi per la professionalità docente restarono comunque separati. Questo elemento è stato sottolineato da Luciana Bellatalla: «Anche in questo caso, dunque, sebbene si debba con soddisfazione salutare una nuova stagione per la formazione insegnante, dobbiamo lamentare il fatto che l'unità della funzione docente non viene riconosciuta» (BELLATALLA, 2009: 90). Si veda anche ULIVIERI, 2006: 42.

${ }^{6}$ In particolare, la riforma voluta dal Ministro Berlinguer, specie nel decreto n. 509 del 3 novembre 1999 , Regolamento recante norme concernenti l'autonomia didattica degli atenei, e la più recente riforma del
} 
sembianze di vere e proprie modificazioni radicali, mentre, in altri, si sono presentate con le fattezze di sottili correzioni, e, sempre, sono state sorrette da un'indomita volontà riformatrice che, seppur in parte lodevole, non è sempre stata oculata ed è risultata spesso scarsamente attenta alle indicazioni provenienti da tutte le voci del mondo accademico. Per le significative differenze che hanno caratterizzato questi due percorsi, se ne proporrà una trattazione particolareggiata e distinta; tuttavia, per comprendere appieno l'impianto comune che li sorreggeva all'interno della legge del 1990, si vogliono far emergere alcuni rilevanti punti di contatto.

\subsection{Centralità delle scienze dell'educazione, università e tirocinio, le nuove coordinate della} formazione insegnante

La legge n. 341 del 1990 è stata emanata dal VI governo Andreotti, il 19 novembre, e pubblicata in Gazzetta Ufficiale il 23 dello stesso mese. Il corso di laurea in Scienze della formazione primaria e la SSIS si collocavano entrambi come specifiche declinazioni, rispettivamente, dei diplomi di laurea ${ }^{7}$ e dei diplomi di specializzazione ${ }^{8}$. Il primo aspetto da rilevare è l'architettura della legge, che inserisce questi percorsi con decisione nell'alveo delle università, rendendoli, a un tempo, uniformi e differenziati rispetto agli altri percorsi. È 1'università, quindi, a essere incaricata del delicato ruolo di gestione della formazione degli insegnanti delle scuole primarie e secondarie e tale formazione sarà svolta anche attraverso il tirocinio, istituendo uno stretto raccordo con il mondo della scuola, che giocherà effettivamente un considerevole ruolo nel successivo svolgimento dei corsi. Il tirocinio è, quindi, un secondo importante punto di contatto tra i due percorsi, che si rivolge al pieno dispiegamento della formazione professionale dell'insegnante.

In entrambi i commi dedicati a questi inediti percorsi, inoltre, si nota l'importante novità del valore abilitante del corso, eliminando quel delicato e controverso momento che era l'esame nazionale di abilitazione, principale canale d'ingresso al mondo della scuola. Infine, i diplomi erano considerati titoli d'ammissione per i concorsi a cattedra. Il valore abilitante dei corsi è, dunque, un altro elemento di vicinanza tra il corso di laurea in Scienze della formazione primaria ${ }^{9}$ e la SSIS $^{10}$.

Ministro Gelmini, Legge 30 dicembre 2010, n. 240, Norme in materia di organizzazione delle università, di personale accademico e reclutamento, nonché delega al Governo per incentivare la qualità e l'efficienza del sistema universitario.

${ }^{7}$ L. 341, 1990, art. 3, comma 2: «Uno specifico corso di laurea, articolato in due indirizzi, è preordinato alla formazione culturale e professionale degli insegnanti, rispettivamente, della scuola materna e della scuola elementare, in relazione alle norme del relativo stato giuridico».

${ }^{8}$ L. 341, 1990, art. 4, comma 2: «Con una specifica scuola di specializzazione articolata in indirizzi, cui contribuiscono le facoltà ed i dipartimenti interessati, ed in particolare le attuali facoltà di magistero, le università provvedono alla formazione, anche attraverso attività di tirocinio didattico, degli insegnanti delle scuole secondarie, prevista dalle norme del relativo stato giuridico».

${ }^{9}$ L. 341, 1990, art. 3, comma 2: «Il diploma di laurea costituisce titolo necessario, a seconda dell'indirizzo seguito, ai fini dell'ammissione ai concorsi a posti di insegnamento nella scuola materna e nella scuola elementare. Il diploma di laurea dell'indirizzo per la formazione culturale e professionale degli insegnanti della scuola elementare costituisce altresì titolo necessario ai fini dell'ammissione ai concorsi per l'accesso a posti di istitutore o istitutrice nelle istituzioni educative dello Stato. I concorsi hanno funzione abilitante».

${ }^{10}$ L. 341, 1990, art. 4, comma 2: «L'esame finale per il conseguimento del diploma ha valore di esame di Stato ed abilita all'insegnamento per le aree disciplinari cui si riferiscono i relativi diplomi di laurea. I diplomi 
Questo aspetto, unitamente all'accesso a numero chiuso e programmato sulle esigenze previste per la futura copertura di posti a cattedra, desiderava porre in continuità e rendere interdipendenti i momenti della formazione e del reclutamento, cercando, se non di risolvere, almeno di porre un argine all'annoso problema del precariato nell'ambito scolastico. ${ }^{11}$

Un ultimo elemento rilevante da porre in luce consiste nella centralità che, almeno a livello intenzionale, le scienze dell'educazione avrebbero dovuto avere nel percorso delineato per la formazione insegnante. Anche in questo caso, ci troviamo di fronte a una stessa scelta operata per il corso di laurea in Scienze della formazione primaria (L. 341, 1990, art. 3, comma 3) e per la SSIS (L. 341, 1990, art. 4, comma 3). Si parla di intenzione, poiché la legge in oggetto non specifica i contenuti dei corsi, ma ne demanda l'attivazione a due successivi decreti del Presidente della Repubblica (DPR), che ne dovevano tracciare le tabelle e specificare modalità e contenuti. Questi importanti elementi di contatto tra i due percorsi per la formazione insegnante non hanno comportato, tuttavia, né una medesima ricezione negli ambiti scientifici né una storia comune. In particolare, proprio questa scelta di posticipare l'emanazione dei regolamenti che soli avrebbero permesso la reale attivazione dei corsi, ha palesato negli anni successivi tutte le difficoltà e le resistenze interne al mondo universitario, coinvolto, proprio in quegli anni, come si è detto, in un profondo processo di trasformazione. Gli attesi regolamenti, infatti, vennero emanati dall'allora Ministro della pubblica istruzione, dell'università e della ricerca scientifica e tecnologica, Luigi Berlinguer, solo il 26 maggio del 1998, con il DM. 153 (pubblicato in GU il 3 luglio), recante il titolo Criteri per l'attivazione dei corsi di laurea in scienze della formazione primaria e scuole di specializzazione per l'insegnamento nelle scuole secondarie.

\subsection{La Scuola di specializzazione per l'insegnamento secondario (SSIS)}

Si ritiene utile iniziare le analisi distinte di questi due percorsi di formazione insegnante dalla SSIS, che tra i due è stato quello che ha vissuto una storia più breve ma decisamente tormentata. Il DM n.153 del 1998, nell'Art. 4 e nell'allegato C, definiva con chiarezza il profilo a venire della formazione insegnante delle scuole secondarie. La SSIS era suddivisa in indirizzi, comprensivi ognuno di una pluralità di classi di abilitazione e ogni Scuola ne avrebbe dovuti attivare quantomeno due. Le aree nelle quali si articolava il curriculum biennale della SSIS erano quattro: area 1 - formazione per la funzione docente; area 2 - contenuti formativi degli indirizzi; area 3 - laboratorio con specifico riferimento ai contenuti formativi degli indirizzi; area 4 tirocinio. Come si può osservare, questo DM rappresentava un'importante sfida, ${ }^{12}$ poiché cercava di definire gli obiettivi formativi per la funzione docente, pur lasciando ampia autonomia ai singoli atenei nella declinazione di questi. Si deve ricordare, infatti, che in precedenza le tabelle ministeriali

rilasciati dalla scuola di specializzazione costituiscono titolo di ammissione ai corrispondenti concorsi a posti di insegnamento nelle scuole secondarie».

${ }^{11}$ Lodevole intenzione che, però, si è scontrata con forti incertezze nel perseguire con decisione questo obiettivo e con ripensamenti viepiù numerosi. Sulla difficile situazione del precariato nella scuola italiana, si veda anche: BRANCATISANO, 2010.

${ }^{12}$ Sfida salutata positivamente da un'ampia parte del mondo scientifico: «I Criteri del 1998 sono un documento pregevole e rappresentano il frutto delle ricerche e delle esperienze che in Italia e all'estero si erano svolte in tema di prima formazione degli insegnanti» (SANTONI RUGIU e SANTAMAITA, 2011: 170). 
regolavano i singoli corsi di studio fornendo una spiccata omogeneità ai percorsi d'istruzione compiuti nei differenti atenei italiani. Con l'abrogazione di dette tabelle, gli obiettivi, approfonditi ma più ampi e generali, hanno assunto la vitale funzione di unificare i percorsi dei futuri insegnanti della scuola secondaria, così come dei laureati nelle singole aree disciplinari. Ciò conferma quella stretta relazione, su cui si tornerà, tra riforma del sistema d'istruzione superiore e rilancio della formazione insegnanti.

Vediamo nel dettaglio in cosa consistevano le aree della SSIS. L'area 1 era dedicata a quelle attività didattiche finalizzate all'acquisizione delle necessarie attitudini e competenze nelle scienze dell'educazione e in altri aspetti trasversali della funzione docente. Dette attitudini e competenze erano oggetto di un'attenta opera di dettaglio nell'allegato A del DM, presentandosi come ambiziose, seppure, come ogni indicazione di cornice, rischiassero di essere davvero molto ampie, disegnando un profilo docente assai complesso e, forse, più volto a una sua rappresentazione ideale che a una stringente indicazione per la realizzazione di un corso post-laurea. Bisogna riconoscere, inoltre, che nonostante la bontà del proposito, il vocabolario utilizzato per definire gli obiettivi rispecchiava una precisa e, a volte, compiaciuta visione della funzione docente, che avrebbe favorito l'innescarsi di polemiche non ancora sopite (ISRAEL, 2008: 230-232; LUZZATTO, 2009: 80-82;). L'area 2:

"Comprende attività didattiche finalizzate all'acquisizione di attitudini e competenze di cui all'allegato A, relative alle metodologie didattiche delle corrispondenti discipline, con specifica attenzione alla logica, alla genesi, allo sviluppo storico, alle implicazioni epistemologiche, al significato pratico e alla funzione sociale di ciascun sapere" (DM. 135, all. C)

Si nota, quindi, come l'architettura della SSIS legasse strettamente le competenze disciplinari - acquisite durante il precedente percorso di laurea e rafforzate con una prospettiva focalizzata sull'insegnamento - con le conoscenze proprie delle scienze dell'educazione, articolate nei loro molteplici ambiti disciplinari. Se le prime due aree si presentavano con una veste didattica non lontana dall'impostazione classica dell'università, con lezioni frontali e seminari la cui verifica era demandata a un esame finale, le novità più significative riguardo alla didattica della SSIS possono essere rintracciate nelle successive due aree. Il laboratorio - area 3 - doveva rappresentare un momento d'integrazione tra le conoscenze dell'area 1 e quelle dell'area 2, prevedendo un'azione coordinata tra i docenti di queste e il possibile ausilio di insegnanti delle scuole medie e secondarie superiori. In questo caso, si deve registrare che la realizzazione nelle singole sedi della SSIS non è stata omogenea e ha fatto sì che quest'area, così delicata e di cerniera, fosse a volte non sufficientemente valorizzata, nonostante le fosse attribuito almeno il $20 \%$ dei Crediti Formativi Universitari (CFU, molto prossimi agli ECTS) dell'intero corso biennale. L'area 4, il tirocinio, infine, era visto come lo strumento per integrare, attraverso le esperienze fatte nelle strutture scolastiche, le competenze teoriche e le competenze operative. Ciò era ottenuto attraverso attività coordinate da un docente supervisore, il quale accoglieva il tirocinante all'interno della sua aula, rendendolo agente attivo di un progetto formativo dettagliato e cadenzato in ogni suo snodo. Si può intuire quanto fosse importante tale ultima area, alla quale doveva essere dedicato almeno il $25 \%$ dei CFU dell'intero corso, per costruire una circolarità tra gli apprendimenti conseguiti e le competenze formate nelle altre e, allo stesso tempo, come essa potesse saldare il mondo della formazione insegnante, interno all'ambito accademico, con l'universo della scuola. Non è 
sorprendente, quindi, che proprio su tale momento della SSIS sia fiorita un'ampia letteratura (LANEVE, 1999; ULIVIERI, GIUDIZI, GAVAZZI, 2002; GENOVESE, 2005a; GENOVESE, 2005b; BONDIOLI et al., 2006; LUSSU, MELONI, 2010) e molti studiosi vi abbiano visto "il momento più alto della formazione dell'insegnante" (SANTONI RUGIU e SANTAMAITA, 2011: 172).

La realizzazione della SSIS nelle diverse sedi regionali nelle quali si sono programmati e svolti i corsi è stata ovviamente differenziata, poiché questi sono stati istituiti in regime di autonomia. Purtroppo, si deve sottolineare l'assenza di un monitoraggio puntuale di questo così significativo tentativo di formazione docente, poiché, già nel 2001, l'allora Ministro dell'Istruzione, dell'Università e della Ricerca, Letizia Moratti, ha sospeso l'azione intrapresa di concerto dalla CRUI (Conferenza dei Rettori) e dalla CONCURED (Conferenza dei centri universitari per la ricerca educativa e didattica) precedentemente istituita proprio per registrare lo svolgimento delle $\operatorname{SSIS}^{13}$. Tale atto, da un lato, palesa una diffidenza sulla quale è necessario soffermarsi e, dall'altro, ha prodotto un polarizzarsi dei giudizi su questa esperienza di cui si vuol dar conto. È indubbio, infatti, che la SSIS sia nata in un preciso snodo della storia del sistema d'istruzione italiano, nel quale un Ministro come Berlinguer ha ridisegnato il mondo universitario (PALOMBA, 2008) e ha inciso profondamente su quello della scuola, legando a doppio filo le sue riforme con una visione specifica dell'istruzione e della cultura. Inoltre, nonostante la prima istituzione della SSIS risalga al 1990, una parte dei decisori politici successivi a Berlinguer, specie coloro che occuparono lo stesso dicastero nei tre governi Berlusconi che succedettero, favorirono una visione di questa esperienza come voluta dalla sinistra italiana (SANTONI RUGIU e SANTAMAITA 2011: 174), avanzando una sospettosa diffidenza e una costante assenza di supporto che ne comportarono prima la sospensione, nel 2008, a opera del Ministro Gelmini, e, poi, l'inevitabile soppressione. Questo atteggiamento di ostilità si è cristallizzato anche nel non rispetto della periodicità triennale dei concorsi a cattedra con i quali sarebbero dovuti essere immessi in ruolo gli abilitati SSIS (LUZZATTO, 2009: 74), periodicità disattesa fin dall'inizio, non essendovi stati concorsi durante tutto il periodo di attività della SSIS.

L'assenza di dati puntuali registrati grazie a un monitoraggio complessivo intrapreso e condotto da organi di governo ${ }^{14}$ ha portato in questi anni a una proliferazione di analisi e di commenti, spesso portati sull'impresa complessiva delle SSIS, i quali, nella stragrande maggioranza dei casi, hanno valutato l'impianto pedagogico e didattico di questa esperienza, rifiutandola o enfatizzandone elementi positivi a seconda della continuità o della distanza rispetto alle posizioni scientifiche degli studiosi ${ }^{15}$. Ciò è stato favorito anche dal convulso momento di rinnovamento del mondo accademico con il quale è coincisa l'attivazione della SSIS, frangente storico che ha visto

\footnotetext{
${ }^{13}$ Sono numerose, invece, le pubblicazioni che hanno monitorato l'operato in senso ampio e aspetti specifici delle singole realtà territoriali della SSIS; queste ricerche rappresentano una ricca miniera di esperienze sulla quale pensare anche le prossime iniziative di formazione insegnante che si stanno svolgendo nell'alveo del Tirocinio Formativo Attivo.

${ }^{14}$ Vi sono, infatti, numerosi studi sulle singole realtà della SSIS, ma è arduo da questi trarne dei giudizi univoci e proporne quindi un bilancio che si possa sottrarre da interpretazioni non solo divergenti ma anche opposte.

${ }^{15}$ Si segnala l'equilibrato tentativo di bilancio condotto da Giunio Luzzatto (LUZZATTO, 2009: 77-80). Si segnala, inoltre, il volume Di Pasqua, Grassilli, Storti (2009).
} 
proprio nel Ministro Berlinguer un importante attore. Accanto a tali trasformazioni, inoltre, occorre ricordare che anche dal punto di vista scientifico, sono numerosi i temi che, seppure non inediti per questi anni, hanno acquisito nuovo slancio in questi due decenni; tra questi, assai rilevante per comprendere alcuni dibattiti nazionali, è il tema della valutazione delle competenze per il profilo della funzione insegnante (GRION e ROBERTS, 2012; PREVITALI, 2012; NIGRIS, 2004; MARGIOTTA, 1999) e il delicato nodo della valutazione delle scuole e degli atenei. Si può concludere che questo significativo esperimento avrebbe avuto bisogno di una maggiore attenzione e, forse, di un clima di valutazione più sereno e che il suo sviluppo sarebbe dovuto essere soppesato con più ponderatezza, senza interrompere così bruscamente il suo percorso, specie in assenza di una valida alternativa per la formazione insegnanti, mancanza che ha pesato sul mondo della scuola fino a oggi, come si avrà modo di vedere.

\subsection{Il corso di laurea in Scienze della formazione primaria}

Il primo anno accademico di attività del corso di laurea in Scienze della formazione primaria fu il 1998/1999, rappresentando una svolta epocale di grande importanza per la formazione degli insegnanti della scuola materna e della scuola elementare (BETTI, 2006). Occorre ricordare, infatti, che fino allora, la formazione di questi si svolgeva, rispettivamente, con un corso di tre anni e con uno di quattro, entrambi a livello di scuola secondaria. Il passaggio in seno all'istruzione superiore, quindi, si presentò come una mutamento assai rilevante, ma fu inevitabilmente non privo di alcuni ostacoli e incertezze: i corsi di laurea, infatti, furono attivati presso le facoltà di Scienze della formazione, eredi delle precedenti facoltà di Magistero ${ }^{16}$, ma ne fu possibile l'istituzione anche in altre facoltà che rispondevano a specifici requisiti. Come nel caso delle SSIS, però, l'attivazione così tardiva di questo corso di laurea costituì uno scarto in confronto alla revisione degli ordinamenti dell'istruzione superiore che, proprio negli stessi anni, vide l'adesione dell'Italia al modello proposto dal Processo di Bologna, implementando un'architettura sorretta da una laurea di primo livello di tre anni e da una di secondo livello di due. In questo modo, la laurea a ciclo unico di quattro anni in scienze della formazione primaria, pensata quasi dieci anni prima, si trovò sfalsata e atipica rispetto a quasi tutti gli altri percorsi d'istruzione superiore ${ }^{17}$.

Il corso di laurea prevedeva un primo biennio comune e un secondo biennio differenziato a seconda dei due indirizzi nei quali si $\operatorname{articolava}^{18}$ : uno per la scuola materna e l'altro per la scuola elementare. La scelta per uno dei due indirizzi era compiuta dallo studente alla fine del secondo anno di corso ed era data la possibilità ai laureati in un indirizzo di acquisire la laurea nell'altro, integrando la propria formazione con non più di due semestri di studio. È rilevante, inoltre, l'organicità con la quale questo corso di laurea dialogava con gli altri, di primo livello o di secondo livello, che potevano essere significativi per la professione insegnante, come ad esempio i corsi di laurea in Scienze dell'educazione: i laureati di questi, infatti, potevano acquisire la laurea in Scienze della formazione primaria a fronte di un puntuale riconoscimento dei CFU acquisiti, in non più di

\footnotetext{
${ }^{16}$ La trasformazione delle Facoltà di Magistero in Facoltà di Scienze della Formazione, fu sancita da un decreto ministeriale del 2 agosto 1995.

${ }^{17}$ Insieme a Giurisprudenza che conserva ancora l'impianto quadriennale. Per un quadro dell'evoluzione normativa del corso di laurea in Scienze della formazione primaria, si veda: Luzzatto (2001).

${ }^{18}$ Il riferimento legislativo è sempre al DM n. 153 del 1998.
} 
quattro semestri integrativi. Un altro elemento d'interesse è la continuità del corso di laurea in Scienze della formazione primaria con l'abilitazione al sostegno per bambini portatori di handicap. Oltre alle attività necessarie ad acquisire la laurea, infatti, potevano essere previste specifiche attività didattiche aggiuntive, per almeno 400 ore, attinenti l'integrazione scolastica degli alunni in situazione di handicap, al fine di acquisire quei contenuti formativi in base ai quali il diploma di laurea poteva costituire titolo per l'ammissione ai concorsi per l'attività didattica di sostegno. Anche in questo caso, un ruolo cardine era ricoperto dal tirocinio finalizzato a esperienze nel settore del sostegno, al quale erano dedicate almeno 100 ore di quelle già previste per quest'area di attività. Chi aveva già conseguito la laurea nel corso, infine, poteva integrare il percorso già compiuto con uno o due semestri aggiuntivi indirizzati al sostegno.

Come e, comprensibilmente, in maniera ancora più marcata rispetto ai programmi della SSIS, il corso di laurea in Scienze della formazione primaria era risolutamente imperniato sulle discipline proprie delle scienze dell'educazione, mantenendo il taglio interdisciplinare caratterizzante della complessità del profilo insegnante. Le aree previste erano quattro: area 1 formazione per la funzione docente; area 2 - contenuti dell'insegnamento primario; area 3 laboratorio; area 4 - tirocinio. L'area 1 rimandava organicamente agli stessi obiettivi contenuti nell'allegato A, che si è visto centrale per la SSIS, cercando di avvicinare e rendere omogenei i percorsi di formazione degli insegnanti a prescindere dalle differenze inerenti al grado della scuola a cui erano rivolti. In tal senso, l'area 1 comprendeva attività didattiche finalizzate all'acquisizione delle necessarie attitudini e competenze nel campo pedagogico, metodologico-didattico, psicologico, socio-antropologico, igienico-medico, nonché relative all'integrazione scolastica per allievi in situazione di handicap. A quest'area era riservato almeno il $20 \%$, per l'indirizzo per la scuola elementare, e almeno il 25\%, per la scuola materna, dei CFU complessivi del corso. L'area 2, centrale nella formazione degli insegnanti, era deputata all'integrazione delle conoscenze proprie delle scienze dell'educazione con attività didattiche finalizzate all'acquisizione di attitudini e competenze in relazione ai fondamenti disciplinari e alle capacità operative nei campi linguisticoletterario, matematico-informatico, delle scienze fisiche, naturali ed ambientali, della musica e della comunicazione sonora, delle scienze motorie, delle lingue moderne, storico-geografico-sociale, del disegno e di altre arti figurative. A quest'area era riservato almeno il $35 \%$, per l'indirizzo per la scuola elementare, e almeno il 25\%, per la scuola materna, dei CFU complessivi del corso. Come nel caso della SSIS, grande enfasi fu posta sulle attività del laboratorio e del tirocinio, a cui erano dedicati almeno il $10 \%$ e almeno il $20 \%$ rispettivamente dei CFU complessivi del corso. È significativo, d'altronde, che l'inizio del tirocinio fosse previsto già dal primo anno di corso, creando piena consapevolezza nella percezione dello studente di quella proficua circolarità tra $\mathrm{i}$ saperi e la loro concreta attuazione e sperimentazione così preziosa nell'ambito delle scienze dell'educazione.

Se l'istituzione e l'attivazione di questo importante corso di laurea devono essere salutati positivamente, bisogna tuttavia segnalare una grave incertezza che ne ha contraddistinto i primi anni (BETTI, 2006: 36). A differenza di quello che era previsto dalla legge del 1990, infatti, il DM del 1998 non aveva attribuito valore abilitante a questo percorso di laurea, incidendo in modo 
significativo sulla valore attrattivo di questo. È stato necessario attendere il $2003^{19}$, per veder attribuito il valore abilitante al corso di laurea in Scienze della formazione primaria, momento che ne ha segnato, nonostante l'intempestività rispetto alla conclusione del primo quadriennio, un rilancio, anche a livello d'iscrizioni, e una sua immagine più definita e compiuta. Di recente, il corso di laurea è passato da quattro a cinque anni, allineandosi, pur nel mantenimento del ciclo unico, alla durata complessiva degli altri corsi universitari di primo e secondo livello.

\section{DALLE SSIS AL TIROCINIO FORMATIVO ATTIVO (TFA)}

\subsection{La riforma del 2010}

Come si è visto nelle parti precedenti, occorre distinguere la formazione iniziale dei docenti della scuola dell'infanzia e primaria (maestri) da quella dei docenti della scuola secondaria di primo e secondo grado (professori). Questa dicotomia permane anche nel recente Decreto 249 del 10 settembre $2010^{20}$, che pure afferma 1' "unitarietà della funzione docente", basata sulla comune "acquisizione di competenze disciplinari, psico-pedagogiche, metodologico-didattiche, organizzative e relazionali necessarie a far raggiungere agli allievi i risultati di apprendimento previsti dall'ordinamento vigente", nonché "delle competenze necessarie allo sviluppo e al sostegno dell'autonomia delle istituzioni scolastiche".

Per quanto riguarda i maestri, il decreto istituisce il corso di laurea quinquennale a ciclo unico, che sostituisce il corso di laurea quadriennale attivato a partire dal 1998-99, mantenendo comunque una sostanziale continuità con esso ${ }^{21}$. Il corso di laurea prevede 600 ore di tirocinio, e la prova finale, che comprende sia la discussione di una tesi sia quella della relazione finale di tirocinio, e rilascia un titolo abilitante per l'insegnamento.

Per quanto riguarda i professori, invece, ci sono importanti novità: il decreto prevede infatti che la loro formazione si realizzi ora in due fasi diverse e successive: la prima, di durata biennale, all'interno di corsi di laurea magistrale appositamente istituiti, ai quali si accede con il possesso della laurea triennale; la seconda, di durata annuale, nel 'Tirocinio Formativo Attivo' (TFA) dopo il conseguimento della laurea magistrale secondo la scansione complessiva $3+2+1$.

Questo provvedimento mira fra l'altro a rendere meno lungo il percorso di formazione rispetto ai sette anni previsti dalla SSIS, prevedendo già a livello di laurea magistrale dei percorsi specifici per i futuri insegnanti secondari, che uniscano preparazione disciplinare e preparazione

\footnotetext{
${ }^{19}$ La Legge di riferimento è la n. 53 del 28 marzo 2003, dal titolo: Delega al Governo per la definizione delle norme generali sull'istruzione e dei livelli essenziali delle prestazioni in materia di istruzione e formazione professionale, esattamente all'Art. 5, comma d.

${ }^{20}$ Decreto 10 settembre 2010, n. 249. Regolamento concernente: Definizione della disciplina dei requisiti e delle modalità della formazione iniziale degli insegnanti della scuola dell'infanzia, della scuola primaria e della scuola secondaria di primo e secondo grado, ai sensi dell'articolo 2, comma 416, della legge 24 dicembre 2007, n. 244.

${ }^{21}$ A giudizio di Giunio Luzzatto (LUZZATO, 2011b), già coordinatore nazionale delle SSIS, la laurea quinquennale abilitante in Scienze della Formazione Primaria, a differenza del TFA, conserva anche nel nuovo Regolamento la sua «nicchia di corretta integrazione tra tematiche educative, discipline, tirocinio scolastico».
} 
professionale. Tuttavia l'istituzione di queste nuove lauree magistrali non è ancora avvenuta, mentre dal corrente anno accademico 2012-2013, a distanza di quattro anni dalla soppressione delle SSIS, è stato attivato il 'Tirocinio Formativo Attivo' (TFA), al quale dunque si accede ancora con il titolo di laurea tradizionale.

Questo contributo tratterà dunque essenzialmente della formazione dei professori, e specificamente del Tirocinio Formativo Attivo, che costituisce in questo momento la principale innovazione attuata nel sistema italiano.

\subsection{Come e perché nasce il TFA}

L'esperienza delle SSIS, la cui durata era di due anni, si è conclusa definitivamente nell'anno accademico 2008-2009 a conclusione del IX Ciclo. Le SSIS sono state sostituite a partire dall'anno accademico 2011-2012 (ma di fatto 2012-2013) dal Tirocinio Formativo Attivo (TFA) di durata annuale.

Il nuovo sistema di formazione iniziale, voluto dal governo di centro-destra, si differenzia dal modello SSIS soprattutto per tre aspetti:

- La minore durata (un anno anziché due).

- Il ridimensionamento della componente socio-psico-pedagogica in favore di quella culturale e disciplinare. ${ }^{22}$

- Il rilevante peso del tirocinio "attivo", fatto direttamente in classe, con l'assistenza di un docente tutor.

Su questa decisione ha pesato, a giudizio di alcuni studiosi, la convinzione, diffusa nel centro-destra, che le SSIS fossero in qualche misura culturalmente (e politicamente) egemonizzate dalla sinistra politica e pedagogica. Di qui la riduzione del peso e della durata della formazione 'professionale' post-lauream rispetto a quella 'culturale', fornita da una università percepita dal centro-destra come politicamente meno schierata (SANTONI RUGIU e SANTAMAITA, 2011).

Altri hanno intravisto nel rafforzamento della formazione culturale dei docenti, soprattutto di quelli di scuola secondaria, un estremo tentativo di frenarne la decadenza puntando gentilianamente su docenti più preparati e perciò anche più capaci di essere buoni insegnanti ("Chi sa, sa anche insegnare"). Tentativo vano, secondo Adolfo Scotto Di Luzio, che pure denuncia la progressiva "corrosione" del modello gentiliano, perché antistorico (SCOTTO DI LUZIO, 2007: 12).

La decisione del centro-destra di sostituire le SSIS con il TFA si spiega tuttavia anche tenendo conto del fatto che per anni, dal loro varo nel 2001, i neoabilitati SSIS avevano avuto la possibilità di iscriversi alle graduatorie permanenti degli abilitati, utili ai fini dell'immissione in ruolo senza concorso sul 50\% dei posti disponibili. Ciò aveva a giudizio del centro-destra gonfiato a

\footnotetext{
${ }^{22}$ Tesi sostenuta in particolare da Giorgio Israel (ISRAEL, 2008), chiamato dal ministro Gelmini a presiedere la commissione per la revisione della formazione iniziale degli insegnanti.
} 
dismisura le graduatorie, creando aspettative che il sistema non avrebbe potuto soddisfare (nel frattempo il Ministero del Tesoro aveva tagliato il Budget di quello dell'Istruzione per 8 miliardi di euro in tre anni, costringendolo a ridurre gli organici $)^{23}$.

Anche il governo di centro-sinistra presieduto da Romano Prodi (2006-2008, ministro dell'istruzione Giuseppe Fioroni) si era posto il problema e aveva deciso di chiudere il rubinetto delle graduatorie permanenti stabilendo che gli ultimi ad avere diritto ad iscriversi nelle graduatorie permanenti sarebbero stati gli specializzandi SSIS dell'VIII Ciclo (Anni Accademici 2006-2007 e 2007-2008): si puntava a bloccare l'ulteriore espansione delle graduatorie e a rivedere l'intera materia del reclutamento (l'ultimo concorso, come già ricordato, era stato bandito nel 1999). Perciò il governo decise per decreto legge (Legge finanziaria 2007) di trasformare le graduatorie permanenti provinciali degli insegnanti abilitati aspiranti all'insegnamento in graduatorie ad esaurimento, con l'obiettivo di assumere in tre anni 150.000 precari.

Quest'ultimo intento fu bloccato dal centro-destra per le citate ragioni di bilancio, ma nella fase di passaggio dal governo Prodi a quello Berlusconi (dalla metà del 2008) il IX Ciclo SSIS (anni 2007-2008 e 2008-2009) partì ugualmente, e i suoi iscritti, che pure non avrebbero potuto inserirsi nelle graduatorie a esaurimento, in seguito a vari ricorsi ottennero alla fine la riapertura una tantum delle graduatorie.

Contestualmente il nuovo ministro dell'istruzione, Mariastella Gelmini, dispose la sospensione del X Ciclo SSIS (Anni Accademici 2008-2009 e 2009-2010) annunciando il proposito di volerlo sostituire con il TFA.

\subsection{Principali caratteristiche del TFA}

Il Tirocinio Formativo Attivo (TFA), introdotto con Decreto Ministeriale nel novembre $2011^{24}$, è istituito dalle università, è attivato per ciascuna classe di abilitazione e attribuisce, tramite un esame finale, il titolo di abilitazione all'insegnamento in una delle classi di abilitazione previste dalla normativa vigente (DM 39/1998 e DM 22/2005), fino a quando tali decreti non saranno sostituiti. Il TFA consiste di tre gruppi di attività:

- Insegnamenti di materie psico-pedagogiche e di scienze dell'educazione (ambiti disciplinari: Pedagogia generale e sociale, Storia della pedagogía, Didattica e pedagogia speciale; Pedagogia sperimentale; Discipline sociologiche e antropologiche) per un totale di $78 \mathrm{CFU}$

- Tirocinio svolto a scuola sotto la guida di un insegnante tutor, comprendente una fase osservativa e una fase di insegnamento attivo (durata: 475 ore).

- Insegnamenti di didattiche disciplinari che vengono svolti stabilendo una stretta relazione tra l'approccio disciplinare e l'approccio didattico.

${ }^{23} \mathrm{Si}$ giunse a sostenere che il ministro dell'istruzione Gelmini era stata "commissariata" da quello dell'economia Tremonti (NICEFORO, 2010, pp. 89-93).

${ }^{24}$ Pubblicato nella Gazzetta Ufficiale n. 288 del 12 dicembre 2011 
- Laboratori pedagogico-didattici indirizzati alla rielaborazione e al confronto delle pratiche educative e delle esperienze di tirocinio.

A tal fine nei laboratori si attua una stretta collaborazione tra docenti universitari, che li dirigono, e insegnanti tutor. L'attività di tirocinio nella scuola si conclude con l'elaborazione di una relazione di tirocinio di cui è relatore un docente universitario e correlatore l'insegnante tutor che ha seguito l'attività di tirocinio.

L'esame finale è sostenuto davanti a una commissione composta da 3 docenti universitari (o dell'alta formazione artistica, musicale e coreutica) che hanno svolto attività nel corso di tirocinio, da 2 tutor o tutor coordinatori, da un rappresentante designato dall'ufficio scolastico regionale ed è presieduta da un docente universitario o dell'alta formazione designato dalla istituzione di riferimento. La commissione assegna:

- Fino a un massimo di 30 punti all'attività svolta durante il tirocinio.

- Fino a un massimo di 30 punti alla prova orale (esposizione orale di un percorso didattico su un tema scelto dalla commissione.

- Fino a un massimo di 10 punti alla discussione sulla relazione finale di tirocinio.

L'esame di tirocinio è superato se il candidato consegue una votazione maggiore o uguale a $50 / 70$.

\subsection{Accesso al TFA}

Per accedere al TFA occorre superare un test preliminare su base nazionale che mira a verificare le conoscenze disciplinari relative alle materie oggetto di insegnamento di ciascuna classe di concorso e le competenze linguistiche di lingua italiana.

Il test è costituito da 60 quesiti a risposta multipla (con quattro risposte, fra le quali il candidato deve individuare l'unica esatta). 10 quesiti sono volti a verificare le competenze in lingua italiana (comprensione di uno o più testi scritti). Gli altri 50 quesiti sono inerenti alle discipline oggetto di insegnamento della classe di concorso. La risposta corretta a ogni domanda vale 0,5 punti, la risposta non data o errata 0 punti. Il test ha la durata di tre ore. Per essere ammesso alla prova scritta il candidato deve conseguire una votazione nel test preliminare non inferiore a 21/30.

La prova scritta è stabilita dalle università e viene superata se il candidato consegue un risultato non inferiore a 21/30. Le università devono attenersi ai seguenti criteri:

- L'oggetto della prova concerne una o più discipline comprese nella classe di concorso cui il percorso di TFA si riferisce.

- La prova verifica le conoscenze disciplinari, le capacità di analisi, interpretazione e argomentazione, il corretto uso della lingua italiana e non può pertanto prevedere domande a risposta chiusa.

- Nel caso di classi di concorso relative alla lingua straniera, la prova è svolta nella lingua straniera scelta dal candidato. 
- Nel caso di classi di concorso che contemplano l'insegnamento della lingua italiana, è prevista comunque una prova di analisi del testo.

- Nel caso di classi di concorso che contemplano l'insegnamento delle lingue classiche è prevista comunque una prova di traduzione.

- Nel caso di classi di concorso relative a discipline scientifiche o tecniche, la prova scritta può essere integrata da una prova pratica in laboratorio. In questo caso il voto è unico ed è ottenuto dalla media aritmetica dei voti attribuiti nella prova scritta e nella prova di laboratorio, ciascuno dei quali deve essere comunque non inferiore a 21/30.

La prova orale, anch'essa gestita dalle singole università, è valutata in ventesimi, e viene superata da chi consegue un risultato non inferiore a 15/20. Nel caso di classi di concorso relative alla lingua straniera, la prova è svolta nella lingua straniera per cui si richiede l'accesso al TFA, nel caso di classi di abilitazione affidate al settore dell'alta formazione artistica, musicale e coreutica la prova orale può essere sostituita da una prova pratica.

La graduatoria finale si ottiene sommando i punteggi conseguiti dai candidati che hanno superato il test preliminare con votazione non inferiore a $21 / 30$, la prova scritta con votazione non inferiore a $21 / 30$ e la prova orale con votazione non inferiore a 15/20. A questi si aggiunge il punteggio attribuito all'esito della valutazione dei titoli.

\subsection{Tutor coordinatori e tutor dei tirocinanti}

La normativa vigente prevede che per lo svolgimento delle attività di tirocinio le università si avvalgano di personale docente e dirigente in servizio nelle istituzioni scolastiche, da utilizzare in qualità di tutor coordinatori o tutor dei tirocinanti. I tutor coordinatori svolgono i seguenti compiti:

- Gestire i rapporti con i tutor degli studenti assegnando questi ultimi alle diverse classi e scuole e formulando il progetto di tirocinio dei singoli studenti.

- Esaminare i materiali di documentazione prodotti dagli studenti nelle attività di tirocinio;

- Supervisionare e valutare le attività del tirocinio diretto e indiretto.

- Seguire le relazioni per quanto riguarda le attività in classe.

I tutor dei tirocinanti hanno il compito di:

- Orientare gli studenti rispetto agli assetti organizzativi e didattici della scuola e alle diverse attività e pratiche in classe.

- Accompagnare e monitorare l'inserimento in classe e la gestione diretta dei processi di insegnamento degli studenti tirocinanti.

I tutor sono designati dai coordinatori didattici e dai dirigenti scolastici delle scuole, iscritte in un apposito elenco, tra $\mathrm{i}$ docenti in servizio con contratto a tempo indeterminato che ne hanno fatto domanda. 


\subsection{Come sta funzionando il TFA}

Nei primi mesi del 2013 sono iniziati i corsi del TFA ordinario, ma in ordine sparso e in modo disomogeneo e confuso.

Alcune università puntano a concludere i corsi con l'esame finale abilitante per maggiogiugno 2013, altre non hanno ancora nominato i tutor coordinatori, anche se il provvedimento per il loro esonero parziale dal'insegnamento è stato finalmente varato.

Il fatto è che il Ministero non ha svolto con efficacia quel lavoro di coordinamento e regia dell'operazione che avrebbe potuto garantire maggiore omogeneità di comportamento tra i diversi Atenei. Al di là dei ritardi sono stati segnalati, per esempio, casi di università che non hanno permesso di svolgere il tirocinio nelle scuole di servizio dei tirocinanti, o che non hanno indicato con esattezza e tempestività né i calendari dei corsi né le prove che dovranno essere sostenute dai corsisti.

\subsection{Il peso della tradizione accademica}

Le università italiane, come si è visto in parte anche con l'esperienza delle SSIS, non sembrano sempre pronte a rendere gli insegnamenti funzionali alle esigenze dei tirocinanti. Favorita in qualche modo anche dalle critiche che erano state formulate alle stesse SSIS (eccessiva enfasi sulla dimensione pedagogica rispetto a quella culturale) vengono proposti in non pochi casi insegnamenti con forte impianto accademico.

Tali insegnamenti sono spesso assai simili a quelli già sostenuti durante gli studi universitari, con scarsa attenzione per le modalità di gestione didattica dei contenuti disciplinari. Considerazioni analoghe valgono, in taluni casi, anche per i corsi dell'area trasversale, malgrado la normativa $^{25}$ vigente prescriva che gli insegnamenti siano "svolti stabilendo una stretta relazione tra l'approccio disciplinare e l'approccio didattico".

Il fenomeno riguarda soprattutto i docenti universitari che hanno avuto scarsi o nessun contatto con il mondo della scuola, essendosi dedicati alla ricerca e alla carriera universitaria subito dopo la laurea. In questi casi una più stretta collaborazione con docenti scolastici esperti sembra poter correggere gli eccessi di "accademismo", ma non sempre questa collaborazione viene cercata o offerta.

\subsection{I laboratori disciplinari e pedagogici-didattici}

Il citato decreto $249 / 2010$ richiede $^{26}$ che vengano apprestati appositi laboratori disciplinari e pedagogici-didattici, ma risulta che in non pochi casi essi non siano stati organizzati adeguatamente, pur essendo, insieme ai tirocini, decisivi per la qualità delle competenze da far acquisire ai corsisti TFA.

${ }^{25}$ Art. 10 comma 3, lettera c del DM 249/2010.

${ }^{26}$ Art. 10, comma 3, lettere c e d. 
Alla conduzione dei laboratori, che dovrebbero operare come veri e propri luoghi di apprendimento pratico della didattica d'aula, sono addetti i tutor coordinatori, ai quali però spesso mancano la capacità e l'esperienza necessarie per esercitare al meglio questa funzione.

\section{IL TFA "SPECIALE"}

Per completare il quadro della formazione iniziale dei docenti in Italia occorre accennare anche a una seconda tipología di TFA, il Tirocinio Formativo Attivo "speciale", che una recente (ma non ancora attuata) disposizione ministeriale ha affiancato al TFA ordinario.

L'ammissione al TFA ordinario, attualmente in corso di svolgimento, è avvenuta su base selettiva, sottoponendo i candidati a una prova di accesso preliminare nazionale, e successivamente a prove scritte sulle materie oggetto delle diverse classi di concorso, organizzate dalle università o istituzioni di alta formazione (AFAM).

Ma il numero degli interessati ad acquisire l'abilitazione superava di molto la disponibilità di posti (circa 20.000), comprendendo anche molti docenti 'precari' con elevata anzianità di servizio - tre anni e più, circa 75.000 - che con l'appoggio dei sindacati e di quasi tutti i partiti politici hanno ottenuto dal Ministero dell'Istruzione nel mese di marzo 2013, alla vigilia delle elezioni politiche, un Decreto rettificativo e integrativo del Decreto base, n. 249/2010.

Il nuovo Decreto ha affiancato al TFA ordinario un altro percorso abilitativo riservato a questi altri aspiranti all'abilitazione, il TFA "speciale", scaglionandolo in tre annualità (2012-13, 2013-14 e 2014-15) sulla base dell'esito di una apposita prova di acceso non selettiva ma vincolante per quanto riguarda la distribuzione nei tre anni e notevolmente influente sul risultato finale (35 punti su 100).

La prova prevede 70 quesiti a risposta multipla (quattro risposte, di cui una sola corretta), così ripartiti:

- Capacità logiche: 30 quesiti.

- capacità di comprensione del testo: 30 quesiti.

- Lingua straniera: 10 quesiti.

Il decreto stabilisce peraltro che i punteggi delle abilitazioni conseguite nei percorsi ordinari saranno diversi e superiori rispetto a quelli delle abilitazioni conseguite nei percorsi riservati.

La decisione del ministro Profumo (ora sostituito nel nuovo governo Letta da Maria Chiara Carrozza) di avviare i TFA speciali ha però suscitato le proteste sia dei corsisti del TFA ordinario, che temono la concorrenza dei molto più numerosi interessati ai TFA speciali, sia di questi ultimi per lo scaglionamento nel tempo e il minor punteggio attribuito.

Comunque mentre i TFA ordinari hanno buone possibilità di concludersi entro pochi mesi, i TFA speciali devono ancora essere organizzati, e non è detto che il nuovo ministro non modifichi le decisioni del suo predecessore Profumo. 
Alla fine di aprile 2013 restano ancora da definire le procedure di raccolta delle candidature per via telematica, gli eventuali raggruppamenti di classi di concorso omogenee e le modalità attuative della prova di valutazione delle competenze in ingresso. Le proteste sono molte, e resistenze vengono anche dalle università che temono di non poter reggere l'ulteriore onere di 75.000 nuovi abilitandi, sia pure ripartiti in tre anni. Un numeroso gruppo di professori universitari (oltre 800 alla fine di aprile 2013), attualmente impegnati come docenti nel TFA ordinario, guidato da due autorevoli esperti di politiche educative come Giunio Luzzatto (università di Genova) e Andrea Ichino (università di Bologna) ha sottoscritto un appello inviato ai Ministri dell'Istruzione (quello precedente Francesco Profumo, e quello nuovo, Maria Chiara Carrozza) a non dare attuazione al TFA “speciale". Nell'appello si legge che "Le università italiane, che negli scorsi mesi sono state chiamate ad organizzare una rigorosa selezione, in quanto centri di sapere e di promozione del merito, non possono prestare il fianco ad un provvedimento che, a nostro parere, tradisce gli stessi principi su cui la selezione si è fondata e i diritti di chi ha seguito le regole sottoponendosi ad un regolare concorso" ${ }^{27}$ : un chiaro riferimento ai corsisti attualmente impegnati nel TFA ordinario.

\section{REFERENCIAS BIBLIOGRÁFICAS}

BARBAGLI, M. e DEI, M. (1969): Le vestali della classe media. Ricerca sociologia sugli insegnanti (Bologna, Il Mulino).

BERLINGUER, L. (2001) (con Marco Panara): La scuola nuova (Bari, Laterza).

BETTI, C. (2006): La formazione universitaria dei maestri, in G. DI BELLO (a cura di), Formazione e società della conoscenza. Storie, teorie, professionalità. Atti del Convegno di Studi, Firenze, 9-10 novembre 2004, pp. 29-40 (Firenze, Firenze University Press).

BONDIOLI, A. et al. (2006): I saperi del tirocinio: formare gli insegnanti nelle SSIS (Milano, FrancoAngeli Editore).

BRANCATISANO, V. (2010): Una vita da supplente: lo sfruttamento del lavoro precario nella scuola pubblica italiana (Milano, Nuovi Mondi).

DI PASQUA, S., GRASSILLI, B. e STORTI, A. (a cura di) (2009): L'eredità della SSIS. «Luci e ombre» della scuola per la formazione degli insegnanti. Atti degli incontri di Trieste e di Bologna, novembre 2008 (Trieste, EUT).

GENOVESE, L. (2005a): La formazione dell'insegnante secondario tra teoria e pratica. Riflessioni e proposte per il tirocinio (Roma, Armando Editore).

GENOVESE, L. (a cura di) (2005b): Insegnanti in formazione: progettare e monitorare il tirocinio (Roma, Armando Editore).

GRION, V. e ROBERTS, S. (2012): Valutazione/autovalutazione degli insegnanti. Approcci, pratiche, strumenti (Padova, CLUEP).

ISRAEL, G. (2008): Chi sono i nemici della scienza? Riflessioni su un disastro educativo e culturale e documenti di malascienza (Torino, Lindau).

LANEVE, C. (a cura di), (1999): Il tirocinio e le professioni educative (Lecce, Pensa Multimedia).

27 Il testo dell'appello può
http://www.adiscuola.it/adiw_brevi/?p=9864.

Revista Española de Educación Comparada, 22 (2013), 139-163

ISSN: 1137-8654 
LUSSU, M.L. e MELONI, S. (a cura di) (2010): Il supervisore di tirocinio nella formazione dell'insegnante. Dieci anni di attività della SSIS di Cagliari (Cagliari, CUEC Editrice). LUZZATTO, G. (2001): Insegnare a insegnare. I nuovi corsi universitari per la formazione dei docenti $-2^{\text {a }}$ ed. (Roma, Carocci).

LUZZATTO, G. (2009): Formazione iniziale: l'Italia al ribaltone, in F. FRABBONI e M.L. GIOVANNINI (a cura di), Professione insegnante. Un concerto a più voci in onore di un mestiere difficile, pp. 67-83 (Milano, FrancoAngeli editore).

LUZZATTO, G. (2011a): La formazione e il reclutamento dei docenti, in V. CAMPIONE e F. BASSANINI (a cura di), Istruzione bene comune. Idee per la scuola di domani (Firenze, Passigli Editori).

LUZZATTO, G. (2011b): Università e scuola per la formazione degli insegnanti, in A.M. AJELLO e V. GHIONE (a cura di), Comunicazione e apprendimento tra scuola e società. Scritti in onore di Clotilde Pontecorvo (Roma, Infantiae.Org).

MARGIOTTA, U. (a cura di) (1999): L' insegnante di qualità: valutazione e performance (Roma, Armando Editore).

NICEFORO, O. (2010): Da Berlinguer a Gelmini. La scuola che (non) cambia (Roma, Editoriale Tuttoscuola).

NIGRIS, E. (a cura di) (2004): La formazione degli insegnanti: percorsi, strumenti, valutazione (Roma, Carocci).

PALOMBA, D. (1988): Scuola e società in Italia nel secondo dopoguerra (Roma, Edizioni dell'Ateneo).

PALOMBA, D. (a cura di) (2008): Changing Universities in Europe and the "Bologna Process" (Roma, Aracne editrice).

PALOMBA, D. (2009): Education and state formation in Italy. In R. Cowen and A. Kazamias (Eds.), International handbook of comparative education, 2 voll., pp. 195-215 (Dordrecht Heidelberg - London - New York: Springer).

PONTECORVO, C. (a cura di) (1979): Università e formazione continua degli insegnanti (Firenze, la Nuova Italia).

PREVITALI, D. (2012): Come valutare i docenti (Brescia, La Scuola).

SANTONI RUGIU, A. e SANTAMAITA, S. (2011): Il professore nella scuola italiana dall'Ottocento a oggi (Roma-Bari, Editori Laterza).

SCOTTO DI LUZIO, A. (2007): La scuola degli italiani (Bologna, Società editrice il Mulino).

ULIVIERI, S. (2006): La formazione degli insegnanti della Secondaria e l'esperienza della SSIS, in G. DI BELLO (a cura di), Formazione e società della conoscenza. Storie, teorie, professionalità. Atti del Convegno di Studi, Firenze, 9-10 novembre 2004, pp. 41-58 (Firenze, Firenze University Press).

ULIVIERI, S., GIUDIZI, G. e GAVAZZI S. (a cura di) (2002): Dal banco alla cattedra: didattica e tirocinio formativo per l'insegnamento nella scuola secondaria (Pisa, Edizioni ETS).

VANNINI, I. (2012): Come cambia la cultura degli insegnanti. Metodi per la ricerca empirica in educazione (Milano, FrancoAngeli).

VISALBERGHI, A. (1978): Pedagogia e scienze dell'educazione (Milano, Mondadori). 


\section{PROFESIOGRAFÍA}

\section{Donatella Palomba}

Donatella Palomba è professore ordinario di Pedagogia generale nell'Università degli Studi di Roma "Tor Vergata", dove coordina il Dottorato Internazionale di Ricerca in Scienze dell'Educazione. Dal 1992 al 2008 ha fatto parte del Comitato Direttivo della Comparative Education Society in Europe (CESE), di cui è stata Presidente dal 2000 al 2004, ed è attualmente Presidente della SICESE (Sezione Italiana della CESE). Dirige la Collana Studi comparativi in educazione - Comparative Education Studies presso l'Editrice Aracne di Roma, ed è membro del Comitato Scientifico di numerose riviste scientifiche italiane e internazionali. I suoi principali interessi di ricerca riguardano l'educazione comparata, le politiche dell'istruzione con particolare riguardo all'insegnamento superiore, l'istruzione a distanza, l'educazione interculturale. Fra le sue più recenti pubblicazioni, il volume Doctoral Studies in Europe. Traditions, Innovations and Trends (Aracne Editrice, 2012), curato insieme a Carlo Cappa, che raccoglie i risultati di una ricerca internazionale da lei coordinata sul dottorato in Europa. Datos de contacto: E-mail: palomba@uniroma2.it.

\section{Carlo Cappa}

Carlo Cappa è ricercatore dal 2007 nell’Università degli Studi di Roma “Tor Vergata”. Le sue ricerche riguardano la storia dell'educazione e la filosofia del Rinascimento italiano ed europeo. Il suo ultimo volume è L'educazione al crocevia della complessità. Sentieri europei della modernità (Aracne Editrice, 2012). Collabora, inoltre, con la cattedra di Pedagogia Generale e di Educazione Comparata dell'Università degli Studi di Roma "Tor Vergata". Ha curato, assieme a Donatella Palomba, il recente volume Doctoral Studies in Europe. Traditions, Innovations and Trends (Aracne Editrice, 2012). É membro del Collegio dei Docenti del Dottorato Internazionale di Ricerca in Scienze dell'Educazione dell'Università degli Studi di Roma "Tor Vergata" in convenzione con l'Universidad de Granada. Fa parte del Comitato scientifico della Collana Studi comparativi in educazione - Comparative Education Studies di Aracne Editrice, Roma. È membro della Comparative and International Education Society (CESE), della Sezione Italiana della Comparative Education Society in Europe (SICESE), della Comparative and International Education Society (CIES) e del Centro Italiano per la Ricerca Storico-Educativa (C.I.R.S.E.). Datos de contacto: Email: carlo.cappa@uniroma2.it.

\section{Orazio Niceforo}

Laureato in Filosofia nell'università degli studi di Milano, ha insegnato materie letterarie ed è stato preside nelle scuole secondarie superiori. Ha fatto parte di importanti commissioni ministeriali per la riforma dell'istruzione secondaria superiore e per otto anni del Consiglio direttivo del Centro Europeo dell'Educazione (ora Invalsi). Lasciata la scuola è stato per alcuni anni consulente del Ministero dell'istruzione per l'innovazione scolastica, ha insegnato legislazione scolastica e storia della scuola nella Scuola di Specializzazione per l'Insegnamento Secondario di Roma e ha tenuto corsi di 'Sistemi scolastici contemporanei' presso l'università di Roma Tor 
Vergata. Ha pubblicato volumi e saggi sulla storia della scuola italiana degli ultimi trenta anni svolgendo una intensa attività pubblicistica su quotidiani e riviste periodiche. Attualmente è docente nel Dottorato Internazionale in Scienze dell'Educazione dell'università di Roma Tor Vergata ed è vicepresidente della SICESE (Sezione italiana della Comparative Education Society in Europe). È redattore della testata online tuttoscuola.com. Datos de contacto: E-mail: orazio.niceforo@gmail.com.

Fecha de recepción: 5 de mayo de 2013.

Fecha de revisión: 15 de mayo de 2013 y 29 de mayo de 2013

Fecha de aceptación: 15 de junio de 2013. 